\title{
Research on Recycling Strategy of Retailer cooperating with the Third-party in Closed-loop Supply Chain
}

\author{
Hongchun $\mathrm{Hu}^{1,4, a, *}$, Xiaoyu Hu ${ }^{2, b}$, Zhiwei Meng ${ }^{3, c}$ Weitang Zhai ${ }^{4, d}$ \\ ${ }^{1}$ Staff Development Institute of China National Tobacco Corporation, Zhengzhou 450008, China \\ ${ }^{2}$ Business Management department, Tong Ling collage, Tong Ling 244000, China \\ ${ }^{3}$ School of Computer and Information, HoHai University, Nan Jing 210000, China \\ ${ }^{4} \mathrm{HeNan} 863$ Software Incubator. LTD, Zhengzhou 450008, China

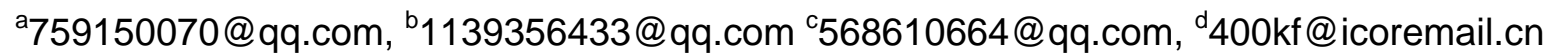

Keywords: closed-loop supply chain; recycling strategy; recycling and reusing; Stackelberg game.

\begin{abstract}
To improve the efficiency of recycling strategies in closed-loop supply chain, the closed-loop supply chain consisting of one manufacturer and one retailer and one third-party is studied. The cooperative recycling model (R3P mode) is designed whereby the retailer collects end-of-life products and the manufacturer cooperates with the third-party firm to handle used products. The two-stage Stackelberg game model of R3P mode is developed under uncertain demand and manufacturer leading. The optimal results are compared with the third-party firm collection model (3P mode). Through the analysis, it is shown that the manufacturer prefers R3P mode when the unit profit of recycling and reusing obsolete products is higher, and the R3P mode's performance is superior to the 3P mode when the third-party firm is a non-profit organization.
\end{abstract}

\section{Introduction}

In the new international situation of global positive response to climate warming, attention to the international energy saving and emission reduction, development of low carbon economy and circular economy, the closed loop supply chain has become a hot research in academic and enterprise. The closed-loop supply chain includes forward and reverse logistics in supply chain [1-2], which can effectively use the resources, alleviating the pressure of environmental pollution. As the world's largest manufacturing country, the development of closed-loop supply chain in China has just started. According to the Ministry of commerce data show that the waste products of only five categories, which are China's color TV, refrigerator, washing machine, air conditioners and computers, has been a breakthrough million in 2011. With the amount of waste products in China have provided a abundance of material basis for the formation of the closed-loop supply chain, but the inefficient recovery strategy causes it is difficult for more and more closed-loop supply chain enterprises to obtain sufficient stable waste products, it is difficult to form a scale effect and the situation of loss is in the majority. Therefore, correct selection of a suitable recovery strategy for increasing the utilization rate of recycling of waste products, and promote the development of closed-loop supply chain is essential

At present, most relevant research results of the closed-loop supply chain mainly concentrated in the operation management, such as the closed-loop supply chain network design, production planning, inventory control. While more and more scholars mainly use the game theory model analysis in the recovery strategy of closed loop supply chain at present. In foreign countries, Savaskan [3] gave the earliest application of non-cooperative game method to analyze the structural problems of bilateral monopoly type recovery strategy of closed loop supply chain. Subramanian [4] analysis the effect of EPR system on the closed-loop supply chain of recycling. Paksoy [5] studied the effects of different modes of transport to the closed-loop supply chain recovery performance and environmental performance. Chen and Chang [10] studied the optimal recovery strategy problem of closed-loop supply chain in cooperation competition condition, obtained that the mode of cooperation OEM 
operators and third party recycling manufacturers competition mode is better than that of OEM and the manufacturer collaborative remanufacturing. Zhou Yongsheng and Wang Shouyang [7] gave the quantitative description of the government monitoring behavior, analysis and comparison of three kinds of recycling mode. Wang Wenbin and Da Qingli [8] discussed the difference between effort and product pricing which is based on the model of a closed-loop supply chain decision under the situation of market subdivision established retailers recycling of waste products and that of the third part. Xiao Fudong [9] studied the effect to closed-loop supply chain on three kinds of recycling channel mode of risk aversion degree of different retailer.

From the existing research results, in the recovery strategy of closed loop supply chain, most analysis the recovery strategy selection and its influence on the performance of closed-loop supply chain, based on the three kinds of recovery strategy which are manufacturers is responsible for recycling, the retailer is responsible for recycling and the third party is responsible for the recovery, and the research consider other types of recovery strategy is relatively less,

So this paper firstly constructed the retailer and the third party collaborative remanufacturing mode as the research which are object closed-loop supply chain in manufacturers, retailers and third party components, then analysis the different between it and the non cooperative remanufacturing mode, and explore the optimal coordinate recovery strategy question of the retailer and the third party, to effectively improve recovery efficiency and the performance of the closed-loop supply chain.

\section{Problem description}

As we known, in the closed-loop supply chain composed of a single manufacturer and a single retailer and single third party recyclers under the uncertain market demand, new products of manufacturers can be completely made from the raw materials, or from part or all of the recycling of waste products, and the quality of both products is no difference.

Based on three basic recovery, this paper design a retailers and the third party coordinate recovery strategy strategies (R3P model) which is shown in Figure 1: the retailer is responsible for recycling waste products, and give the recovery of products to third party, then the third party is responsible for the remanufacturing of waste products, its products are given to manufacturers to process products. In order to analysis the performance of the R3P model, this paper compared and analyzed the different between the R3P model and the 3P mode that the third party is responsible for recycling and remanufacturing. Assume that the unit cost of the manufacturer is $C$, the wholesale price of products is $\mathrm{W}$, the market sales price is $\mathrm{P}$, then the market demand function is $D(p)=a-b p$, where $a>0, b>0$, $a$ is the largest size of the market, $B$ is price sensitive coefficient.

In order to simplify the calculation, the paper only gives static analysis on the use of recycled products, namely the recycling amount $D(p) \theta$, which $\theta(0 \leq \theta \leq 1)$ for recycling rate. According to Savaskan, the recovery investment cost I can be set to $I=c_{R} \theta^{2}$, in which $c_{R}>0$ is investment cost coefficient. It assumes that the unit gains of the remanufacturing of used products of manufacturer or third party are the same, namely G. In the 3P and R3P mode, the manufacturer needs to signed a cooperation agreement with third parties, in which will pay the recovery fixed investment cost to the third parties. the hypothesis is $\varphi=F \theta$, in which $\mathrm{F}$ is the largest transfer payment of manufacturer when the waste products recycling utilization rate reached 1.

\section{Analysis of Recovery Strategies}

Analysis of the 3P mode .In the closed-loop supply chain under the mode of 3P, the manufacturer is in a dominant position, in which according to their own cost, manufacturers determine the products wholesale price $\mathrm{W}$ and the maximum compensation expenses for third party $\mathrm{F}$, and then the retailer who accept the manufacturer's wholesale price will determine the market selling price $\mathrm{P}$, third party decided the recycling rate $\theta$ according to the manufacturer's compensation. So the profit of manufacturers, retailers and the third party respectively:

$$
\Pi_{M}^{3 P}=(a-b p)(w-c)+g \theta(a-b p)-F \theta
$$




$$
\begin{aligned}
& \Pi_{R}^{3 P}=(a-b p)(p-w) \\
& \Pi_{3 P}^{3 P}=F \theta-c_{R} \theta^{2}
\end{aligned}
$$

The 3P decision problem model can be used to establish the two stage manufacturer dominated game model. According to backward induction method of the Stackelberg game theory, firstly to seek the the first order conditions for P to (2) and $\frac{\partial^{2} \Pi_{R}^{3 p}}{\partial p^{2}}=-2 b<0$, we can conclude that $p_{3 P}^{*}=\frac{a+b w}{2 b}$. In the same way as $\frac{\partial^{2} \Pi_{3 P}^{3 P}}{\partial \theta^{2}}=-2 c_{R}<0$, with seeking the the first order conditions for $\theta$ to (3), we can conclude that $\theta_{3 P}^{*}=\frac{F}{2 c_{R}}$. Then we fed $p_{3 P}^{*}$ and $\theta_{3 P}^{*}$ into (1):

$$
\max _{w, F} \Pi_{M}^{3 P}=\frac{1}{2}\left(w a-b w^{2}-c a+c b w\right)+\frac{1}{4 c_{R}}\left(F g a-F g b w-2 F^{2}\right)
$$

Because $0<\theta_{3 P}^{*}<1$, so $16 c_{R}>g^{2} b+g(a-b c)$. At the same time, $0<\theta_{3 P}^{*}<1$, then $16 c_{R}>g^{2} b+g(a-b c)$. While $a-b c>0$, so $16 c_{R}>g^{2} b$. Then the Hessian matrix of (4) is: $\left[\begin{array}{cc}-b & \frac{-g b}{4 c_{R}} \\ \frac{-g b}{4 c_{R}} & \frac{-1}{c_{R}}\end{array}\right]=\frac{b}{c_{R}}-\frac{g^{2} b^{2}}{16 c_{R}^{2}}>0$. It means that $\Pi_{M}^{3 P}$ is a concave function on the $\mathrm{W}$ and $\mathrm{F}$, which there is a maximum. From the first order conditions of (1)-(3), we can get the optimal wholesale price, the optimal compensation expenses, the optimal recycling rate respectively:

$$
\begin{aligned}
& w_{3 P}^{*}=\frac{8 c_{R} a-g^{2} b a+8 c_{R} b c}{16 c_{R}-g^{2} b} \\
& F_{3 P}^{*}=\frac{2 c_{R} g(a-b c)}{16 c_{R}-g^{2} b} \\
& \theta_{3 P}^{*}=\frac{g(a-b c)}{16 c_{R}-g^{2} b}
\end{aligned}
$$

So the manufacturers, retailers and third party optimal profit in the closed-loop supply chain respectively is: $\Pi_{M}^{* 3 P}=\frac{2 c_{R}(a-b c)^{2}}{b\left(16 c_{R}-g^{2} b\right)}, \quad \Pi_{R}^{* 3 P}=\frac{16 c_{R}^{2}(a-b c)^{2}}{b\left(16 c_{R}-g^{2} b\right)^{2}}, \quad \Pi_{3 P}^{* 3 P}=\frac{c_{R} g^{2}(a-b c)^{2}}{\left(16 c_{R}-g^{2} b\right)^{2}}$.

Analysis of the R3P model. In this model, the manufacturer is also in a dominant position as in the mode of 3P. Firstly, we give the wholesale price of $\mathrm{W}$ and compensation expenses of $\mathrm{F}$, then the retailer decides the selling price of $P$ products and recycling of waste product rate, the third party will decide whether to accept the recycling protocol cooperation, and assumed the retailers only recovery the waste products which can be reused, namely $\tau=\theta$. When the waste products are delivered to the third party, retailers can obtain income units for $\mathrm{R}$, and the third party received unit income is g-r from the use of waste products. In R3P mode, the manufacturers, the retailers and the third party profit respectively:

$$
\begin{aligned}
& \Pi_{M}^{R 3 P}=(a-b p)(w-c)-F \theta \\
& \Pi_{R}^{R 3 P}=(a-b p)(p-w)+\theta r(a-b p)-c_{R} \theta^{2} \\
& \Pi_{3 P}^{R 3 P}=F \theta+\theta(g-r)(a-b p)
\end{aligned}
$$

The R3P decision problem model can be used to establish the two stage manufacturer dominated game model. Because $0<\theta_{R 3 P}^{*}<1$, so $4 c_{R}>r(a-b w)+r^{2} b$; and from $a-b w>0$, we get $4 c_{R}>r^{2} b$. Then the Hessian matrix of (9) is: $\left[\begin{array}{cc}-2 b & -r b \\ -r b & -2 c_{R}\end{array}\right]=4 c_{R} b-r^{2} b^{2}>0$, It means that $\Pi_{R}^{R 3 P}$ is a concave function on the $\mathrm{p}$ and $\theta$. From the first order conditions of (9), we can get: $p_{R 3 P}^{*}=\frac{2 c_{R} a-a b r^{2}+2 c_{R} b w}{b\left(4 c_{R}-r^{2} b\right)}, \theta_{R 3 P}^{*}=\frac{r(a-b w)}{\left(4 c_{R}-r^{2} b\right)}$

Proposition 1: When $F \geq-(g-r)\left(a-b p_{R 3 P}^{*}\right)$, the third party willing to accept the cooperation of the R3P mode. 
Proof: By type (10), when $\Pi_{3 P}^{R 3 P} \geq 0$, the third party will accept recycling cooperation, i.e. $F \geq-(g-r)\left(a-b p_{R 3 P}^{*}\right)$.

Proposition 1 can explain the contract condition of the manufacturer and the third party cooperation under R3P mode. When $r \geq g,-(g-r)\left(a-b p_{R 3 P}^{*}\right) \geq 0$. It means that If the units income of the retailer recycling of waste products higher than that of recycling and remanufacturing, manufacturers need to compensate the third party to stimulate the third party willing to engage in remanufacturing. When $r<g,-(g-r)\left(a-b p_{R 3 P}^{*}\right)<0$. It means that even if the manufacturer does not give third party compensation, the third party manufacturing production can reap the rewards in the recovery, and is willing to recycling coordination of R3P mode in closed loop supply chain. In proposition 1,we let $F=\underline{F}+\varepsilon$, in which $\underline{F}=-(g-r)\left(a-b p_{R 3 P}^{*}\right), \varepsilon>0$. We fed $p_{R 3 P}^{*}$ and $\theta_{R 3 P}^{*}$ in (8), and get:

$$
\Pi_{M}^{R 3 P}=\frac{(a-b w)\left(2 c_{R} w-2 c_{R} c-F r\right)}{4 c_{R}-r^{2} b}
$$

And because $\Pi_{M}^{R 3 P}(\underline{F})>\Pi_{M}^{R 3 P}(F)$, so when $F=-(g-r)\left(a-b p_{R 3 P}^{*}\right)$, The $F$ value reaches the minimum, the profit of the manufacturer reaches the maximum value.

When fed $F=-(g-r)\left(a-b p_{R 3 P}^{*}\right)$ into (11), we get $\frac{d^{2} \Pi_{M}^{R 3 P}}{d w^{2}}<0$, From the first order conditions of (8)-(10), we can get the optimal wholesale price, the optimal compensation expenses, the optimal recycling rate respectively:

$$
\begin{aligned}
& w_{R 3 P}^{*}=\frac{4 c_{R} b c-b^{2} c_{R}^{2}-2 a r b g+r^{2} b a+4 c_{R} a}{b\left(8 c_{R}-2 r g b\right)} \\
& F_{R 3 P}^{*}=\frac{c_{R}(r-g)(a-b c)}{4 c_{R}-r g b} \\
& \theta_{R 3 P}^{*}=\frac{r(a-b c)}{8 c_{R}-2 r g b}
\end{aligned}
$$

So the optimal profit of manufacturers, retailers and third party in the closed-loop supply chain in the R3P model respectively is: $\Pi_{M}^{* R 3 P}=\frac{c_{R}(a-b c)^{2}}{2 b\left(4 c_{R}-r g b\right)}, \Pi_{R}^{* R 3 P}=\frac{c_{R}\left(4 c_{R}-r^{2} b\right)(a-b c)^{2}}{4 b\left(4 c_{R}-r g b\right)^{2}}, \Pi_{3 P}^{* R 3 P}=0$.

Comparative analysis. Proposition 2: when $16 c_{R} r-8 c_{R} g+r g^{2} b>0, \theta_{R 3 P}^{*}>\theta_{3 P}^{*}$.

Proof: from (14) and (7), we get $\theta_{R 3 P}^{*}>\theta_{3 P}^{*}$, then $16 c_{R} r-8 c_{R} g+r g^{2} b>0$.

Proposition 3: when $4 r>g, \Pi_{M}^{* R 3 P}>\Pi_{M}^{* 3 P}$.

Proof: we can get $\Pi_{M}^{*_{R} R 3 P}=\frac{c_{R}(a-b c)^{2}}{2 b\left(4 c_{R}-r g b\right)}$ in the model of R3P. let it compare with $\Pi_{M}^{* 3 P}$, we can get $4 r>g$.

According to proposition 2 and 3, when $4 r>g$, it is better to implement R3P model than 3P model for manufacturer, and at the same time, the recovery utilization rate of R3P mode in closed loop supply chain is better than that of 3P model.

Inference: if $\mathrm{r}=\mathrm{g}$, the optimal profit and recovery utilization rate of the R3P model of manufacturers, in the closed-loop supply chain is better than that of 3P model.

Proof: if $\mathrm{r}=\mathrm{g}$, then $4 r>g$. According to proposition 2 and 3 , we get $\theta_{R 3 P}^{*}>\theta_{3 P}^{*}, \Pi_{M}^{* R 3 P}>\Pi_{M}^{* 3 P}$. While if $\Pi^{* R 3 P}>\Pi^{* 3 P}$, then $112 c_{R}-r^{2} b>0$. Because when $\mathrm{r}=\mathrm{g}, 0<\theta_{R 3 P}^{*}<1$ and $0<\theta_{3 P}^{*}<1$, we could get $4 c_{R}>r^{2} b$, which can meet the condition of $112 c_{R}-r^{2} b>0$, so $\Pi^{*{ }^{* 3 P}}>\Pi^{* 3 P}$.

By infer, if the third party as a non-profit organization, uses the R3P model in the closed-loop supply chain, then the closed-loop supply chain performance, manufacturer optimal profits and recycling rate is superior to that of the 3P model, which is consistent with the conclusion of the empirical study of Kumar[10], so the government should encourage and support more third party nonprofit organizations to participate in closed loop supply chain operation, so as to improve the 
performance of the closed-loop supply chain and enhance the level of energy-saving emission reduction of closed-loop supply chain.

\section{Conclusion}

This paper studies the recovery strategy of closed-loop supply chain. In the closed-loop supply chain composing by manufacturers, retailers and third party, designed a recycling cooperative mode in which the retailer is responsible for recycling of waste products, the third party is responsible for recycling and reuse of waste products (R3P model), compared it with the 3P mode in witch the third party is responsible for recycling, then respectively, established two order Stackelberg game model to calculate the optimal decision in closed-loop supply chain of two kinds of mode, through comparative analysis we found that, manufacturers and more inclined to R3P mode, and when the third party as a nonprofit organization, the R3P model is better than 3P model, so the government should encourage and support more third party nonprofit organizations to participate in closed loop supply chain operation, so as to improve the performance of the closed-loop supply chain and enhance the level of energy-saving emission reduction of closed-loop supply chain. The recovery strategy research of the closed loop supply chain in this paper is only consider linear uncertain demand, further in the multi-stage closed-loop supply chain, competition environment and other uncertain research aspects and so on can be researched.

\section{Acknowledgements}

The authors thank the financial support of the 2014 key state projects in scientific research and technological development of the tobacco company of Henan province (HYKJ201414).

\section{References}

[1]Nunen V J, Zuidwijk R A.E-enabled closed-loop supply chains[J]. California Management Review, 2004,46(2):40-54.

[2]Sehultmann F,Zumkeller M,Rentz O. Modeling reverse logistic tasks within closed-loop supply chains: an example from the automotive industry[J]. European Journal of Operational Research, 2006, 171 (3): 1033-1050.

[3]Savaskan R C, Bhattacharya S, Wassenhove L N. Closed-loop supply chain models with product remanufacturing[J]. Management Science,2004, 50(2): 239-252.

[4]Subramanian R, Gupta S, Talbot B. Product design and supply chain coordination under extended producer responsibility[J]. Production and Operations Management,2009,18(3):259-277.

[5]Turan Paksoy, Tolga Bektas, Eren Ozceylan. Operational and environmental performance measures in a multi-product closed-loop supply chain [J].Transportation Research Part E, 2011, 47: 532-546.

[6]Jen-Ming Chen, Chia-I Chang. The coopetitive strategy of a closed-loop supply chain with remanufacturing[J]. Transportation Research Part E, 2012,48:387-400.

[7] Zhou Yong-sheng, Wang Shou-yang. Calling back EOL products under the supervision of the government[J]. Systems Engineering-Theory \& Practice, 2010,30(4):615-621.

[8] Wang Wen-bin, Da Qing-li.The study on collection and pricing for closed-loop supply chain with retailer and the third party collecting[J]. Journal of Industrial Engineering/Engineering Management, 2010,24(2):130-134.

[9]Kumar S, Malegeant P. Strategic alliance in a closed-loop supply chain, a case of maufacturer and eco-non-profit organization. Technovation, 2006,26:1127-1135.

[10]Xiao Fu-dong, Nie Jia-jia, Zhao Dong-mei.Research on collecting strategies in the closed-loop supply chain with risk aversion retailer[J]. Industrial engineering and management, 2011, 16(5): 60-67 